\title{
Development of Laser Induced Breakdown Spectroscopy for studying erosion, deposition, and fuel retention in ASDEX Upgrade
}

\author{
Peeter Paris ${ }^{\mathrm{a}}$, Kaarel Piip ${ }^{\mathrm{a}}$, Antti Hakola ${ }^{\mathrm{b}}$, Matti Laan ${ }^{\mathrm{a}}$, Märt Aints ${ }^{\mathrm{a}}$, Seppo Koivuranta ${ }^{\mathrm{b}}$, Jari Likonen ${ }^{\mathrm{b}}$, \\ Aleksandr Lissovski ${ }^{\mathrm{a}}$, Matej Mayer ${ }^{\mathrm{c}}$, Rudolf $\mathrm{Neu}^{\mathrm{c}}{ }^{\mathrm{d}}$, Volker Rohde ${ }^{\mathrm{c}}$, Kazuyoshi Sugiyama ${ }^{\mathrm{c}}$, ASDEX \\ Upgrade Team $^{\mathrm{c}}$
}

\author{
${ }^{a}$ Institute of Physics, University of Tartu, Tartu, Estonia \\ ${ }^{b}$ VTT Technical Research Centre of Finland, Espoo, Finland \\ ${ }^{c}$ Max-Planck-Institut für Plasmaphysik, Garching, Germany \\ ${ }^{d}$ Technische Universität München, Fachgebt Plasma-Material-Wechelwirkung, Garching, Germany
}

\begin{abstract}
The paper deals with the development of laser induced breakdown spectroscopy (LIBS) into an in situ method for studying erosion/deposition processes at the first walls of fusion reactors. To this end, samples extracted from the divertor tiles of ASDEX Upgrade after the 2009 plasma operations were analyzed using LIBS for their composition and the results were compared with other post mortem deposition data. Quantitative depth profiles for the elemental concentrations were extracted from time-resolved LIBS spectra by applying a novel data processing method. In addition, both multiline and multispot averaging procedures were applied to reduce fluctuations in the data. The LIBS concentration profiles matched qualitatively with those given by secondary ion mass spectrometry and quantitatively with the ion-beam data. The deuterium content of the samples could be reliably determined if the surface densities were $>10^{17} \mathrm{at} / \mathrm{cm}^{2}$.
\end{abstract}

Keywords: plasma-facing components, LIBS diagnostics, elemental depth profiles, fuel retention, data processing.

\section{Introduction}

Laser induced breakdown spectroscopy (LIBS) is a promising method for remote in situ monitoring of erosion/deposition processes and retention of plasma fuel on the first walls of fusion reactors [1]. By recording LIBS spectra induced by successive laser pulses, one can extract the depth profiles of various elements on the surface layers. At the moment, the main open issue in fusion-related LIBS studies is obtaining quantitative information on the concentrations of different elements in the deposited layers.

The standard method would be using different calibration curves [2] but to apply this technique, a large number of pre-calibrated reference samples with similar deposits as on the wall structures of fusion reactors would be needed. Calibration-free LIBS (CF LIBS), based on the so-called Boltzmann plot method [3], does in principle not need calibration curves but it assumes local thermal equilibrium of the LIBS plasma and the plasma properties (e.g. electron temperature $T_{e}$ and density $n_{\mathrm{e}}$ ) have to be known accurately. The slope-ratio method [4] can give a quick picture on the concentrations of composite materials by comparing their LIBS spectra with those extracted from corresponding bulk materials. However, reliable results have been obtained only for samples consisting of 2-3 different elements.

In [5] we carried out LIBS analyses for W-coated Mo samples, exposed to Magnum-PSI plasmas, by recording both in situ and post mortem LIBS spectra as a function of the number of laser shots. Depth profiles for elemental concentrations were successfully obtained by applying a novel data processing method. However, it is not clear whether the method will work in the case of codeposited layers on real tokamak samples. Here we present the results of post mortem LIBS analyses of selected ASDEX Upgrade (AUG) samples The main task is to extract depth profiles for different elements on the surface layers from LIBS measurements and crosscheck the results with those determined by other surface characterization methods. In addition, a detailed discussion on a novel data processing method will be presented.

\section{Experimental}

For the analyses, three different wall tiles were removed from the inner (tile 4) and outer divertor (tiles 1 and 3B-II) of AUG (see Fig. 1) after its 2009 experimental campaign with deuterium plasmas [6]. The entire tiles were first measured using Rutherford Backscattering Spectroscopy (RBS) and Nuclear Reaction Analysis (NRA) after which cylindrical samples (diameter $17 \mathrm{~mm}$, height $10 \mathrm{~mm}$ ) were drilled from them for LIBS and Secondary Ion Mass Spectrometry (SIMS) analyses. All the tiles had originally 1-10 $\mu \mathrm{m}$ thick $\mathrm{W}$ marker coatings on finegrained graphite and also uncoated poloidal stripes at the centre of each tile. As a result of plasma operations, up 
to $1-\mu \mathrm{m}$ thick co-deposited layers consisting mainly $\mathrm{H}$, $\mathrm{D}, \mathrm{C}, \mathrm{B}$, and $\mathrm{W}$ were formed on the tiles.

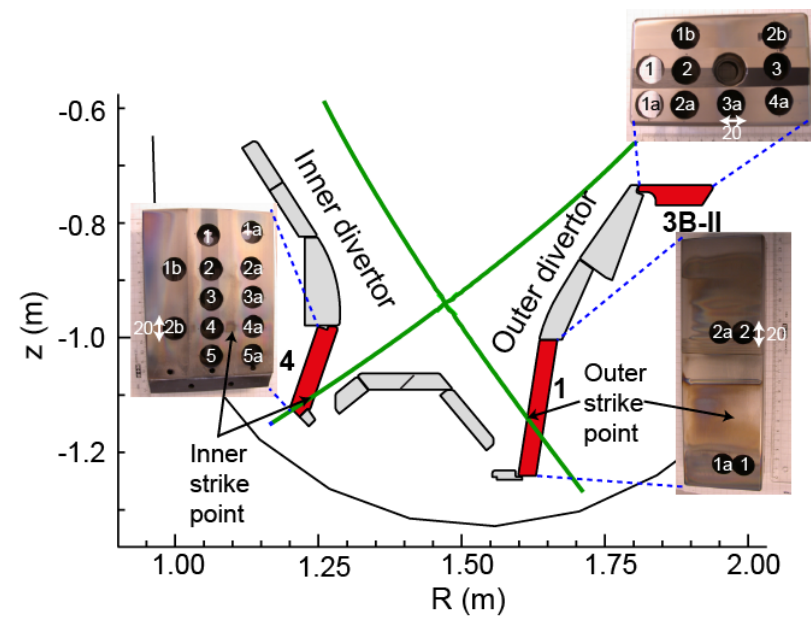

Fig. 1. Poloidal cross section of the AUG divertor in 2009 together with photos of the analyzed tiles (marked in red). The numbering scheme of the samples used in SIMS and LIBS measurements is also shown. The green lines denote the separatrix of a typical plasma discharge.

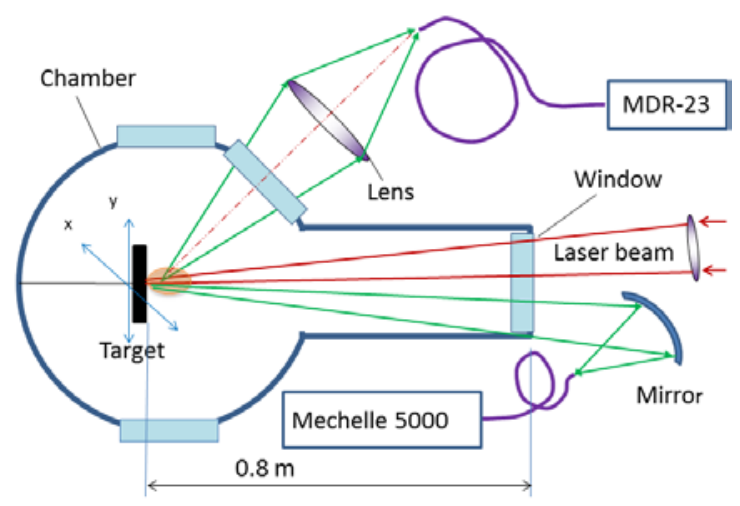

Fig. 2. Schematic illustration of the setup used in the LIBS experiments.

The LIBS measurements were carried out using a setup schematically shown in Fig. 2. The studied samples were located in a vacuum chamber which could be evacuated down to $10^{-6}$ mbar. Using argon at 1 mbar background pressure improved remarkably the signal-tobackground ratio. The LIBS spectra were excited by focussing the radiation of a Nd:YAG laser (Quantel YG980, $\lambda=1064 \mathrm{~nm}$, pulse duration $6 \mathrm{~ns}$ ) onto the sample surface where the laser fluence was $\approx 10 \mathrm{~J} \mathrm{~cm}^{-2}$. The chosen value of the fluence was a compromise between a low value for the ablation rate (to enhance depth resolution of LIBS) and an acceptable signal-tonoise ratio.

The LIBS spectra were recorded simultaneously by two spectrometers. Details of the setups are presented in [7]. The first, the Mechelle5000 recorded the spectrum in the entire 245-850 nm range for every laser shot. This spectrometer was foreseen for the determination of the elemental composition of the samples. The CzernyTurner type MDR-23 spectrometer, for its part, was used in high-resolution measurements in 20-nm wavelength ranges, especially to spectrally resolve hydrogen and deuterium Balmer $\alpha$-lines. Compared with Mechelle, MDR-23 had better signal-to-noise ratio. For timeresolved measurements, the delay between the laser pulse and the beginning of the recording gate, $t_{\mathrm{d}}$ and the width of the gate, $\Delta t$, where optimized to obtain as good signal-to-background ratio for the spectral lines as possible. For Mechelle 5000, the optimal values were $t_{\mathrm{d}}$ $=100 \mathrm{~ns}$ and $\Delta t=1000 \mathrm{~ns}$, while for MDR-23 they were $t_{\mathrm{d}}=200 \mathrm{~ns}$ and $\Delta t=2000 \mathrm{~ns}$.

The LIBS data were compared with those obtained by RBS, NRA and SIMS. The RBS measurements were carried out using 2.5-3.0 MeV protons (scattering angle $165^{\circ}$ ) along the uncoated graphite stripes next to the $\mathrm{W}$ markers to obtain the amount of $\mathrm{W}$ re-deposited on the surface. NRA was used to determine the surface concentrations of $\mathrm{D}, \mathrm{C}$, and $\mathrm{B}$ in the deposited layers. We used 2.5- $\mathrm{MeV}^{3} \mathrm{He}^{+}$ions, and the protons formed in the nuclear reactions $\mathrm{D}\left({ }^{3} \mathrm{He}, \mathrm{p}\right){ }^{4} \mathrm{He},{ }^{11} \mathrm{~B}\left({ }^{3} \mathrm{He}, \mathrm{p}\right){ }^{13} \mathrm{C}$ and ${ }^{12} \mathrm{C}\left({ }^{3} \mathrm{He}, \mathrm{p}\right){ }^{14} \mathrm{~N}$ were recorded at $150^{\circ}$.

Depth profiles of different elements on the marker coatings were measured by SIMS. In the analyses, the VG Ionex IX-70S double-focusing magnetic sector instrument with a 5-keV $\mathrm{O}_{2}{ }^{+}$primary ion beam and with a current of $500 \mathrm{nA}$ was used [9]. The beam was rasterscanned across an area of $0.3 \times 0.4 \mathrm{~mm}^{2}$.

\section{Results and discussion}

\subsection{LIBS spectra}

The thickest deposited layers after the 2009 campaign were measured at the inner divertor, close to the inner strike point. As a consequence, the LIBS measurements will be the most reliable in this region and the following discussion therefore concentrates mainly on sample $2 \mathrm{~b}$ drilled from tile 4 (see Fig. 1). The sample had originally a 1- $\mu \mathrm{m} \mathrm{W}$ coating on graphite.

The LIBS spectra were recorded from 10 different spots on the sample surface, each ablated by 20 laser pulses. The spectra of the first laser pulses were, however, not representative for the analyses because of heavy surface contamination (including water vapor) resulting from sample fabrication and storage phases. For a single spot, the spectrum was noisy and the intensity of spectral lines fluctuated from shot-to-shot, but averaging over 10 spots [10] smoothed remarkably the fluctuations. Fig. 3 compares the single-spot and averaged spectra for sample $2 \mathrm{~b}$ (tile 4 ).

We identified 9 main elements in the Mechelle 5000 spectrum and used the integral intensities of 43 spectral lines in the analysis; for example, carbon was characterized by the average intensity of $10 \mathrm{CII}$ lines. The off-peak background B (see Fig. 3) was first subtracted from the measured signal $S$ and then integrated over the selected peak. 


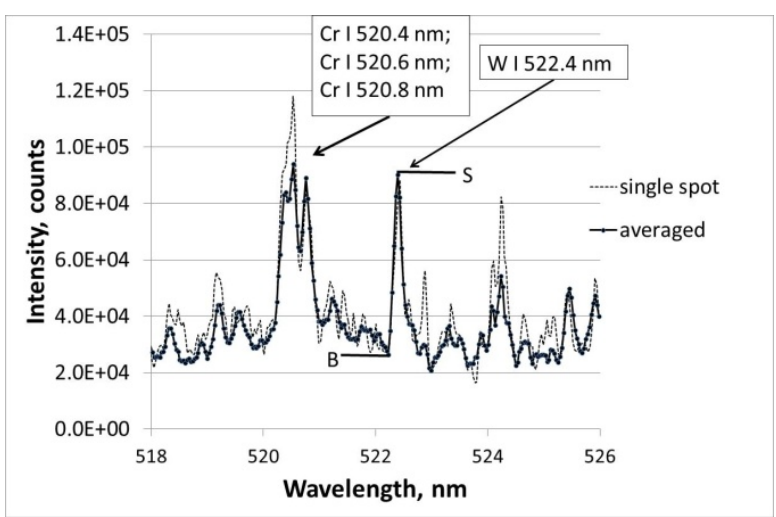

Fig. 3. Part of Mechelle 5000 spectrum around $522 \mathrm{~nm}$ for sample $2 b$, tile 4 and for laser shot \#2. The strongest peaks have been identified in the figure. Here, $\mathrm{B}$ is the background and $\mathrm{S}$ the measured signal.

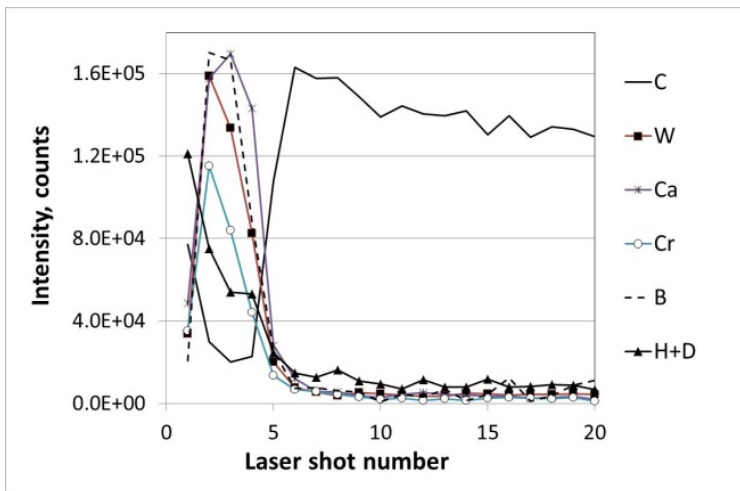

Fig. 4. Integral intensities of selected spectral lines as a function of the laser shot number for sample $2 b$, tile 4 , extracted from the Mechelle 5000 data.

Fig. 4 shows the intensities for 7 elements on sample $2 \mathrm{~b}$ (tile 4) as a function of the laser shot number. Because of unsufficient resolution of the Mechelle spectrometer, only the sum of $\mathrm{H}_{\alpha}$ and $\mathrm{D}_{\alpha}$ is presented. The LIBS profiles show that the deposited material and the $\mathrm{W}$ coating are removed during the first five laser shots. In the case of carbon it is possible to distinguish the deposited layer from the graphite substrate. The strong $\mathrm{Cr}$ and $\mathrm{Ca}$ signals here are due to surface contamination. Notice that these raw data tell nothing about the true concentrations of the elements due to their different sensitivities in the spectra.

Using the MDR-23 spectrometer it was possible to separate the hydrogen and deuterium lines. Figure 5 shows that for sample $2 b$ (tile 4) peaks belonging to $\mathrm{H}_{\alpha}$ and $\mathrm{D}_{\alpha}$ are easily detectable for shots \#2-5. A high $\mathrm{H}_{\alpha}$ peak arising during the first shot is likely caused by water on the surface. Starting from the sixth shot, when the radiation of the substrate (CII peaks) appears, deuterium is not detectable anymore. The persistent but weak hydrogen signal may be caused by impurities of the background Ar gas.

In case of the sample $1 \mathrm{~b}$ from tile 3B-II a reliable detection of deuterium was possible only during the second shot and carbon appeared at shot three. On this sample, the signal levels for $\mathrm{D}_{\alpha}$ were about 3 times lower than those for sample 2b (tile 4). Simultaneously, the number of shots needed to remove the surface layers was two times smaller, indicating that sample $1 \mathrm{~b}$ contains $\sim 6$ times less $\mathrm{D}$ than sample $2 \mathrm{~b}$. This is consistent with NRA data: $9.0-11.9 \times 10^{17} \mathrm{at} / \mathrm{cm}^{2}$ for sample $2 \mathrm{~b}$ and 2.3$2.7 \times 10^{17} \mathrm{at} / \mathrm{cm}^{2}$ for sample $1 \mathrm{~b}$. We can also conclude that in our setup the detection limit for $\mathrm{D}$ is around $10^{17}$ $\mathrm{at} / \mathrm{cm}^{2}$.

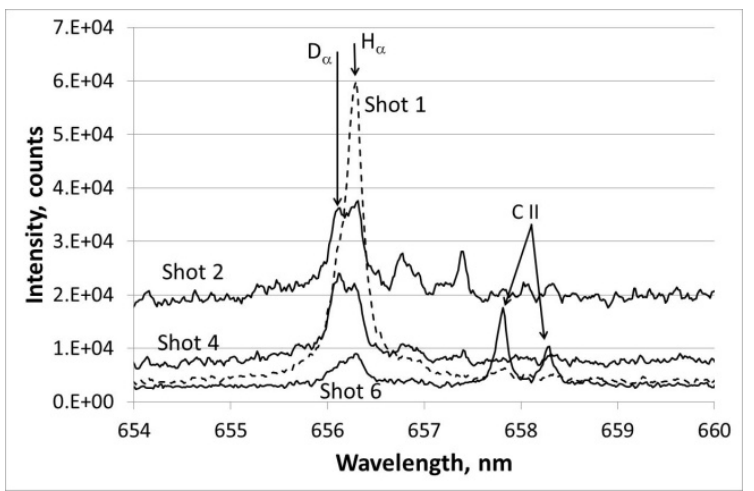

Fig. 5. LIBS spectra around $656 \mathrm{~nm}$ at different laser shots as measured by the MDR-23 spectrometer (sample 2b, tile 4).

\subsection{Determining elemental concentrations}

Fig. 6 shows depth profiles obtained by SIMS for sample 4a (tile 4), which contains similar co-deposits as the sample $2 \mathrm{~b}$. It should be pointed out that compared to LIBS, SIMS has considerably higher spatial resolution: 5 $\mathrm{nm}$ for SIMS, $300 \mathrm{~nm}$ for LIBS between successive measurement points. In SIMS, the detection limits are also approximately 1000 times larger than those in LIBS.

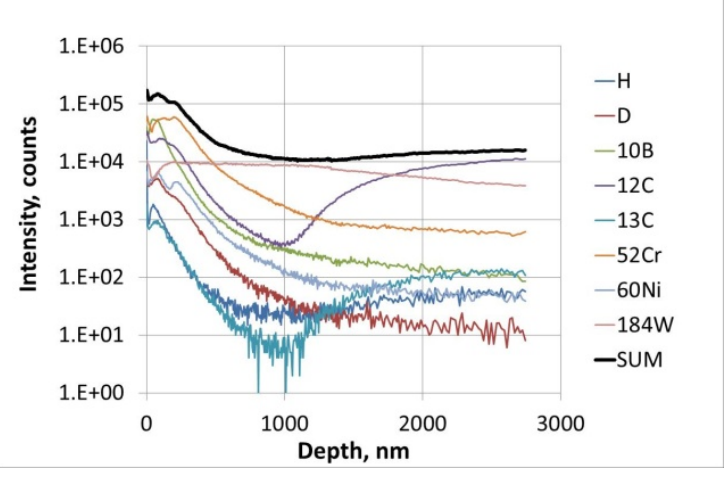

Fig. 6. SIMS depth profiles for sample 4a, tile 4. The thick line at the top is the sum of intensities of all the elements.

By comparing the profiles in Figs. 4 and 6 one finds many signals behaving qualitatively in a similar manner. Conversion to relative concentrations requires, however, more careful data analysis. If neglecting self-absorption of spectral lines and different matrix effects, the intensity of a spectral line should be proportional to the concentration of the corresponding element. Hereinafter we describe a method which allows one to find these proportionality coefficients from the LIBS data.

For each laser shot the sum of relative concentrations of sample elements is equal to one. In an ideal case the sum of relative concentrations over all shots should be equal to the number of shots $N$. 
Type equation here.Because of limited accuracy of measurements this condition holds approximately

$$
\sum_{n}\left[\sum_{m}\left(x_{m} I_{n, m}\right)\right] \approx N
$$

Here $n$ denotes the shot number, $m$ the index of a certain element, $x_{\mathrm{m}}$ the proportionality coefficient to be found and $I_{\mathrm{m}, \mathrm{n}}$ the intensity of $m^{\text {th }}$ element at the $n^{\text {th }}$ shot. According to the least square method the coefficients $x_{m}$ can be found by minimizing the function $S(x)$

$$
S(x)=\sum_{n}\left[\sum_{m}\left(x_{m} I_{m, n}\right)-1\right]^{2}
$$

For this, we require that $\frac{\partial}{\partial x_{m}} S(x)=0$, which leads to the system of linear equations $M X=V$. If $E$ is the number of sample elements and $i, j=1 \ldots E$, then $M_{i, j}=\sum_{n} I_{i, n} I_{j, n}$ and $V_{i}=\sum_{n} I_{i, n}$. Solution of the system gives coefficients $x_{\mathrm{m}}$ and the product $x_{m} I_{m n}$, as a function of the laser shot number $n$ is the depth profile of the concentration of the $m^{\text {th }}$ element.

The depth profiles of relative concentrations obtained by LIBS were compared with the SIMS profiles. To make this possible, the SIMS profiles were normalized by dividing the intensity of a particular element by the sum of all intensities. In the case of LIBS, the conversion of laser pulse number to depth was performed by setting the laser ablation rate to $300 \mathrm{~nm} /$ pulse. The resulting curves are shown in Fig. 7. A good qualitative agreement between the shapes of SIMS and LIBS profiles is obtained. Only for depths $>1500 \mathrm{~nm}$, the $\mathrm{W}_{\text {SIMS }}$ curve decays at a much slower rate than $\mathrm{W}_{\mathrm{LIBS}}$, due to the large surface roughness of the samples. Based on NRA data, the $\mathrm{D} / \mathrm{C}$ ratio on the 1- $\mu \mathrm{m}$ thick surface layer is $~ 1.2-1.4$ and by combining the RBS and NRA results, the D/W ratio becomes $\sim 0.8-1.1$. The correspondence between LIBS and RBS/NRA is therefore relatively good. In the case of $\mathrm{D}$, the $\mathrm{D}_{\text {SIMS }}$ curve is 10 times too small, due to the $\mathrm{D}$ signal being partly outside the electronic gate of SIMS during the measurements.

Testing the limitations of the data processing procedure showed that besides general limitations (signal-to-noise ratio, etc.) [2] the proposed method has some specific caveats. First, it works only when shot-toshot changes in the concentrations exceed a certain level. Secondly, in the case of elements whose LIBS profiles coincide, it is not possible to estimate their concentrations separately.

\section{Conclusion}

The used LIBS setup allowed reliable detection of deuterium in samples extracted from AUG tiles. A method for data processing was also proposed which allowed using the LIBS data for the determination of depth profiles for elemental concentrations in multilayer structures. The method is simple and does not need any information of the laser plasma plume parameters. Relative concentrations show satisfactory quantitative correspondence with those obtained by ion-beam methods.

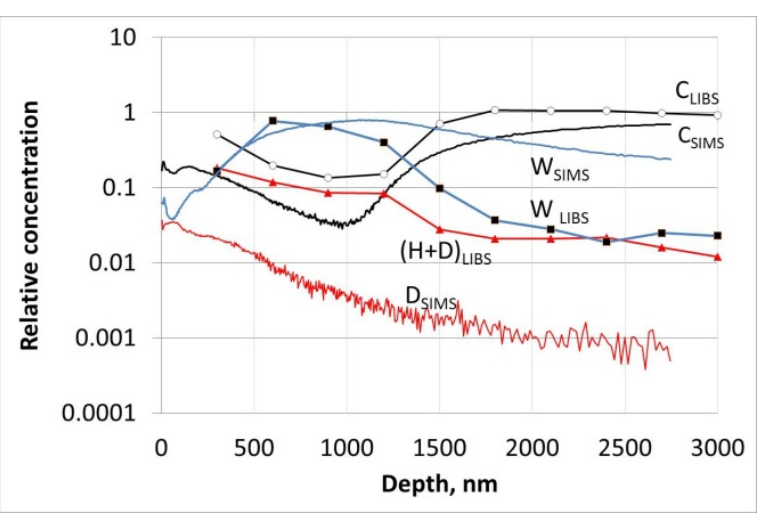

Fig. 7. Comparison of depth profiles of concentrations found by SIMS and LIBS.

\section{Acknowledgments}

This work has been carried out within the framework of the EUROfusion Consortium and has received funding from the Eoropean Union's Horizon2020 research and innovation programme under grant agreement number 633053. . The views and opinions expressed herein do not necessarily reflect those of the European Commission.

\section{References}

[1] V. Philipps et al., Development of laser-based techniques for in situ characterisation of the first wall in ITER and future fusion devices, Nucl. Fusion 53 (2013) 093002.

[2] D. W. Hahn and N. Omenetto, Laser-Induced Breakdown Spectroscopy (LIBS), Part II, Appl. Spectrosc. 66 (2012) 347-419.

[3] B. Gornushkin and U Panne, Radiative models of laserinduced plasma and pump-probe diagnostics relevant to laser-induced breakdown spectroscopy, Spectrochim. Acta Part B 65 (2010)345-259.

[4] A.Malaquias et al, Development of ITER relevant laser techniques for deposited layer characterisation and tritium inventory, J. Nucl. Mater. 438 (2013) 5936-5939.

[5] K. Piip et al, LIBS analysis of tungsten coatings exposed to Magnum PSI ELM-like plasma, 21 ${ }^{\text {st }}$ Int. Conf. PSI, 2014, 26-30 May, Kanazawa, Japan, paper P3-032, submitted to J. Nucl. Mater.

[6] A. Kallenbach et al., Overview of ASDEX Upgrade results, Nuclear Fusion 51 (2011) 094012.

[7] A. Lissovski et al, LIBS for tungsten diagnostics in vacuum: selection of analytes, $21^{\text {st }}$ Int. Conf. PSI, 2014, 26-30 May, Kanazawa, Japan, paper P3-011, submitted to J. Nucl. Mater.

[8] M. Mayer, "SIMNRA Users Guide”, Tech. Rep. IPP (1997) 9/113, Max-Planck-Institut für Plasmaphysik, Garching

[9] A., Hakola et al., Migration and deposition of ${ }^{13} \mathrm{C}$ in the full-tungsten ASDEX Upgrade tokamak Plasma Phys. Control. Fusion 52 (2010) 065006.

[10] P.Paris, et al., Determination of elemental depth profiles by multi-spot averaging technique of LIBS spectra, Fusion Eng. Design 86 (2011) 1125-1128. 